\title{
A Patient With a Zenker's Diverticulum Showing Symptoms Similar to Acute Coronary Syndrome
}

\author{
Keita Oyama ${ }^{\text {a }}$, Nobuhiro Akuzawa ${ }^{\text {a c }}$, Toshihiro Suga ${ }^{a}$, Takashi Hatori ${ }^{\mathrm{a}}$, Kunihiko Imai ${ }^{\mathrm{a}}$, \\ Yonosuke Kitahara $^{\text {a }}$, Masahiko Kurabayashi ${ }^{b}$
}

\begin{abstract}
A 61-year-old man with no significant medical history was admitted to our hospital because of upper chest pain and discomfort. Urgent coronary angiography with possible diagnosis of acute coronary syndrome (ACS) showed normal findings. Further questioning after coronary angiography revealed that patient's symptoms had gotten worse soon after swallowing a piece of sushi without chewing. Computed tomography of the chest revealed air particles outside the posterior hypopharynx wall, and subsequent esophagography showed Zenker's diverticulum (ZD) of about $1 \mathrm{~cm}$ in diameter. The discomfort resolved on day 2. Findings on esophageal manometry and upper gastrointestinal endoscopy showed no remarkable abnormalities, and the entrance of the ZD could not be identified on endoscopic examination. The patient was discharged on day 7 without any complications. Physicians should know that esophageal diseases, including ZD, might show symptoms mimicking ACS, and that not obtaining a detailed clinical history can lead to misdiagnosis.
\end{abstract}

Keywords: Chest discomfort; Computed tomography; Esophagography; Zenker's diverticulum

\section{Introduction}

Esophageal diverticula are rare, and are observed in less than $1 \%$ of upper gastrointestinal X-ray images [1]. Among esophageal diverticula, Zenker's diverticulum (ZD) is commonly observed as a posterior pharyngoesophageal pouch having characteristics of a pseudodiverticulum and consisting of only

\footnotetext{
Manuscript accepted for publication September 17, 2015

${ }^{a}$ Department of Internal Medicine, Social Insurance Gunma Chuo General Hospital, 1-7-13 Koun-cho, Maebashi, Gunma 371-0025, Japan

bepartment of Medicine and Biological Science, Gunma University Graduate School of Medicine, 3-39-22 Showa-machi, Maebashi, Gunma 371-8511, Japan

${ }^{\mathrm{c}}$ Corresponding Author: Nobuhiro Akuzawa, Japan Community Health Care Organization Gunma Chuo Hospital, 1-7-13 Koun-cho, Maebashi, Gunma 371-0025, Japan.Email: akuzawa_nobuhiro@yahoo.co.jp
}

doi: http://dx.doi.org/10.14740/jmc2311w mucosal and submucosal tissue, and is formed by pulsion forces in an area with relative wall weakness between the oblique fibers of the inferior pharyngeal constrictor and the horizontal fibers of the cricopharyngeus $(\mathrm{CP})$ muscles $[1,2]$. $\mathrm{ZD}$ is predominantly seen in men and in older people, and has an estimated overall prevalence of between $0.01 \%$ and $0.11 \%$ [2]. The most common symptom of ZD is dysphagia, accounting for $80-90 \%$ of patients' complaints, and symptoms associated with ZD may be present for weeks to years before a definite diagnosis is made [2]. Dysphagia is relevant to extrinsic compression of the cervical esophagus by the enlarged diverticulum itself in addition to incomplete opening of upper esophageal sphincter (UES) [2]. Patients with ZD may show various symptoms such as odynophagia, dysphonia, hypersalivation, nausea, vomiting, halitosis, choking sensation, cough, and aspiration pneumonia $[2,3]$.

We present here a rare case of ZD with upper chest discomfort as an initial symptom mimicking acute coronary syndrome (ACS) after food boluses transiently lodge in the diverticulum.

\section{Case Report}

A 61-year-old man was admitted to the emergency department of our hospital because of increasing upper chest oppression, which developed after eating lunch. He had felt intermittent dull feeling in the throat and dysphagia while eating 2 weeks before admission. He had had no significant medical history or family history. He was a nonsmoker and did not consume alcohol. On admission, his height was $166 \mathrm{~cm}$, weight was $58 \mathrm{~kg}$, body temperature was $36.1^{\circ} \mathrm{C}$, and blood pressure was $104 / 82$ $\mathrm{mm} \mathrm{Hg}$. His heart rate was 72 beats per minute and was regular. Physical examination revealed no significant abnormalities. The patient presented with persistent chest discomfort, but both his arterial blood $\mathrm{O}_{2}$ saturation (98\%) and partial $\mathrm{O}_{2}$ pressure (92.1 Torr) were normal. Both chest X-ray and electrocardiogram showed normal findings. Laboratory tests findings were also normal, with a white blood cell count of $4,600 / \mathrm{mm}^{3}$

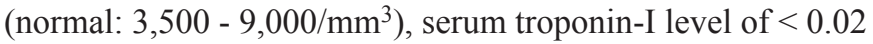
$\mathrm{ng} / \mathrm{mL}$ (normal: $0.02-0.04 \mathrm{ng} / \mathrm{mL}$ ), creatine kinase $(\mathrm{CK})$ level of $93 \mathrm{U} / \mathrm{L}$ (normal: $24-195 \mathrm{U} / \mathrm{L}$ ) and CK-MB level of $<5.0$ $\mathrm{ng} / \mathrm{mL}$ (normal: $<5.0 \mathrm{ng} / \mathrm{mL}$ ). Levels of lipid parameters, including low-density lipoprotein cholesterol, high-density lipoprotein cholesterol, and triglycerides, were normal. Plasma fi- 


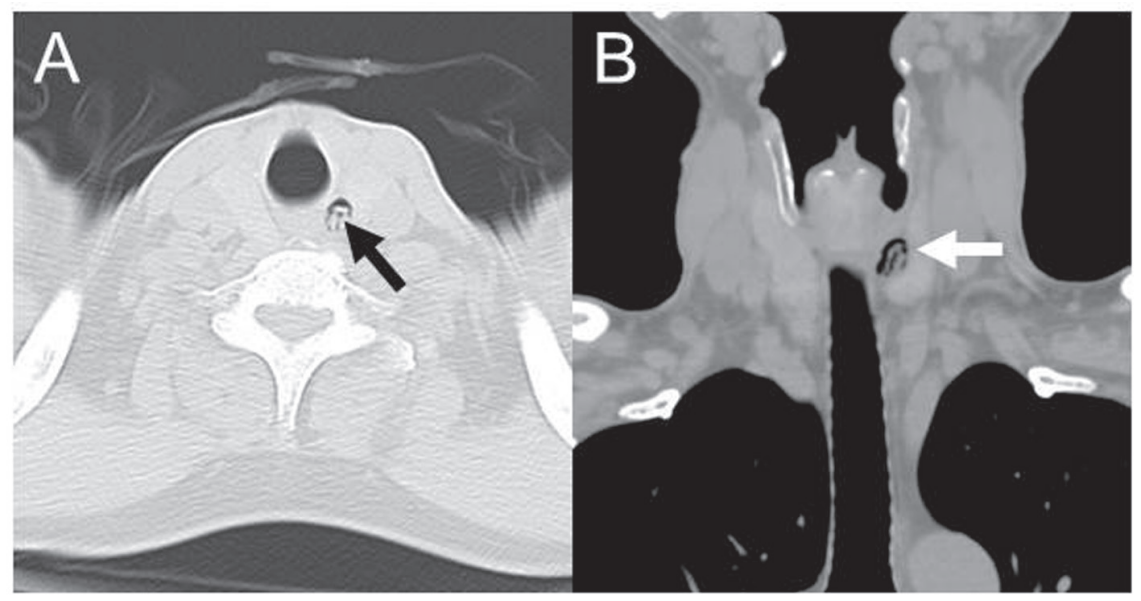

Figure 1. Images of plain computed tomography (CT) of the neck and the chest on admission. (A) Plain axial image of the chest. An air particle containing slightly low-density contents was observed adjacent to left posterior wall of the hypopharynx (black arrow). (B) Coronary CT image of the chest also revealed unilocular air particle with a smooth margin and low-density internal contents (white arrow).

brin/fibrinogen degradation products and D-dimer levels were also normal. Transthoracic echocardiography did not show massive pericardial effusion or other obvious abnormalities. Because sublingual nitroglycerin did not improve the patient's chest discomfort, emergency coronary angiography (CAG) was performed via the right femoral artery using a 6-Fr sheath after treatment with oral aspirin $(200 \mathrm{mg})$, oral clopidogrel (300 mg), and intravenous heparin (5,000 IU). However, CAG showed normal findings; no stenotic or occlusive lesions were found in the left or right coronary arteries. Further questioning after CAG revealed that the patient's upper chest discomfort had developed soon after eating sushi topped with a piece of avocado. Furthermore, the patient had swallowed a piece of sushi whole. Based on this information, plain computed tomography (CT) of the neck, the chest, and the upper abdomen was performed to search for esophageal foreign bodies, and revealed an air particle containing low-density contents in an area between the left lobe of the thyroid gland and the hypopharynx (Fig. 1A, B). This suggested the possibility of left hypopharynx wall perforation resulting from swallowing a piece of sushi without chewing.

Soon after performing the plain chest CT, we also consulted otolaryngologists in our hospital regarding the risk of mediastinitis after hypopharynx wall injury. The otolaryngologists pointed out that ZD might exist in the position of the observed air particle because the lesion had a smooth and round outline, had internal low-density contents probably due to food residue, and was located just above the UES where ZD commonly develops. We decided to observe the patient's progress under fasting conditions in our hospital and started the administration of infusion solution $(1,500 \mathrm{~mL} /$ day $)$ and piperacillin sodium (6 g/day), considering the risk of mediastinitis. The patient's chest discomfort gradually ameliorated after admission and completely disappeared on day 2 . In accordance with the advice of otolaryngologists, esophagography using watersoluble contrast was performed on day 3 . Esophagography revealed a diverticulum with a narrow neck and maximum diam- eter of about $1 \mathrm{~cm}$ protruding from the left side of the cervical esophagus (Fig. 2), leading to a definite diagnosis of ZD. There was also a filling defect inside the diverticulum, probably due to food debris, as with CT findings on admission. On day 4, examination of the inside of the hypopharynx, esophagus,

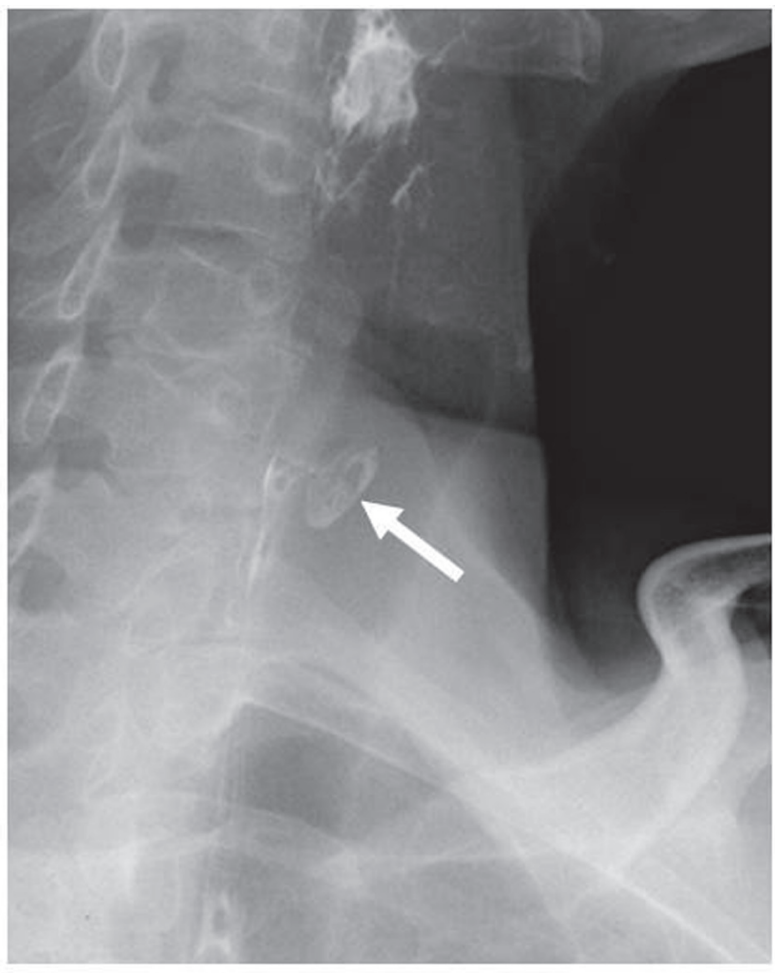

Figure 2. Image of esophagography using water-soluble contrast on day 3. Esophagography revealed a diverticulum (white arrow) with a narrow neck and maximum diameter of about $1 \mathrm{~cm}$, protruding from the left-posterior side of the cervical esophagus. The diverticulum located at the height of the sixth cervical vertebra. 
stomach and duodenum using an endoscope was performed, but there were no remarkable abnormalities and the entrance of the ZD could not be identified. The laboratory findings on day 5 were completely normal and the general status of the patient was good; esophageal manometry on day 5 showed no significant abnormalities. Administration of piperacillin was terminated on day 5 , and meals were started on day 6 . The patient did not complain of any symptoms, including dysphagia, while eating. Contrast-enhanced CT of the chest was done on day 7 and showed no significant abnormalities except for findings associated with ZD. We recommended that the patient be followed up regularly in an outpatient clinic because we could not directly observe the contents of ZD endoscopically, and thus we could not exclude the possibility that neoplastic lesions might exist inside ZD. The patient was discharged from our hospital on day 7, but did not visit our hospital again after discharge. Subsequently, the patient was lost to follow-up.

\section{Discussion}

$\mathrm{ZD}$ is commonly observed in a proximal position to the esophagus, and occurs in a location above the UES composed of the posterior surface of the thyroid gland, cricoid cartilage and three muscles: the inferior pharyngeal constrictor, the $\mathrm{CP}$ muscles and cervical esophagus [1]. The most widely accepted mechanism for the development of $\mathrm{ZD}$ is a functional disturbance of the hypopharynx, including increased resting pressure of the UES, lack of sufficient relaxation, and lack of coordination between the hypopharynx and the UES [1]. Patients with ZD may show dysfunction of the UES opening, replacement of the $\mathrm{CP}$ muscle fibers by fibrous adipose tissue, or a significant increase of the collagen to elastin ratio, leading to increased hypopharyngeal pressure due to decreased compliance of these muscles $[1,4]$. Spastic condition of the CP muscle may also contribute to the onset of ZD [1]. Esophagography is still the mainstay of definite diagnosis of ZD and can determine its size and location [4]. Dynamic continuous fluoroscopy is necessary for evaluating regurgitation of undigested food collected in ZD and the resultant aspiration [2]. Endoscopy has limited value in the diagnosis of ZD [1]; however, endoscopic examination of $\mathrm{ZD}$ is necessary to rule out ZD-associated cancer, which may develop as a result of chronic irritation and inflammation due to food or liquid stasis [4]. Endoscopic examination is also useful for evaluating regurgitation of swallowed material, known as post-swallow hypopharyngeal reflux (PSHR), which is highly sensitive and specific for ZD [5], though our case did not show PSHR. ZD may be identified by transcutaneous ultrasonography of the neck as a mass lesion mimicking a thyroid mass and showing an increase in the lesion's size during ingestion of water [2]. The usefulness of chest CT for the diagnosis of ZD is unclear. Gorkem et al [6] reported a pediatric case in which chest CT findings of a large esophageal pouch filled with food and air particles led to definite diagnosis of ZD. In another case, multiple examinations by chest CT could not identify a large ZD (4 cm in diameter) [7]. In our case, an air particle in the left retrohypopharyngeal space on CT was the decisive factor in considering the possibility of $\mathrm{ZD}$. Accordingly, physicians must not miss unusual air particles adja- cent to the hypopharynx on $\mathrm{CT}$, which suggest the possibility of ZD.

Symptoms associated with ZD may be present for weeks to years before its definite diagnosis [2]. However, the trigger mechanism by which asymptomatic ZD changes into symptomatic ZD is unclear. Commonly, the size of ZD is strongly related to the appearance of the various symptoms; small diverticula may not cause symptoms, but larger diverticula are usually symptomatic and patients with very large diverticula may show a gurgling swelling that is palpable in the neck [4]. In the present case, the ZD was about $1 \mathrm{~cm}$ in diameter and therefore relatively small. The patient had not noticed any symptoms at all before admission. The patient had undergone upper gastrointestinal endoscopy annually for 10 years prior to admission, but no endoscopists identified ZD. Incidental or iatrogenic perforation of undiagnosed ZD during endoscopic procedure or nasogastric tube insertion has been reported [4]. Therefore, although ZD is a rare disease, physicians should know that ZD might be found in asymptomatic patients who have regularly undergone periodic examinations of the upper gastrointestinal tract. Regarding correlation between the size of ZD and symptoms, Bergeron et al [8] reported that PSHR is predictive of a ZD larger than $1 \mathrm{~cm}$. They classified 49 patients with ZD into three groups according to the diameter of their ZD lesions: small $(<1 \mathrm{~cm})$, medium $(1-3 \mathrm{~cm})$ and large $(>3 \mathrm{~cm})$. Interestingly, they reported that over $80 \%$ of the patients with small ZD had already shown solid dysphagia, which was more common than liquid dysphagia. Conversely, about $70 \%$ of the patients with medium or large ZD had complained of symptoms associated with food or liquid regurgitation. Similarly, PSHR was seen in $90.9 \%$ and $86.7 \%$ of patients with medium and large ZD, respectively, compared with $16.7 \%$ of patients with small ZD. These results suggest that patients with ZD larger than $1 \mathrm{~cm}$ may show symptoms resulting from both dysphagia and regurgitation, whereas most patients with small ZD may only have dysphagia or difficulty in swallowing. Therefore, in patients with small ZD, it should be necessary to determine the cause of dysphagia before definite diagnosis of ZD, such as oropharyngeal dysphagia and esophageal dysphagia due to neuromuscular diseases or obstructive lesions [9]. Among diseases causing esophageal dysphagia, esophageal motility disorders resulting from hypercontractility or spasticity are reported to produce recurrent, angina-like and retrosternal chest pain of non-cardiac origin [10]. Furthermore, a case of esophageal perforation by a foreign body presenting with severe chest pain similar to ACS has also been reported [11]. Although the limitations of our report include our inability to perform endoscopic examination of the ZD on admission and a lack of follow-up information after discharge, deterioration of upper chest discomfort observed in our case on admission may associate with food residue temporarily stuck in the ZD because patient's symptoms got worse and persistent soon after swallowing a piece of sushi without chewing. Accordingly, it should be noted that esophageal diseases might show symptoms mimicking ACS and a lack of questioning on detailed clinical history can easily lead to misdiagnosis. Moreover, although it is rare, physicians should be aware that complications of ZD, including diverticulitis, peptic ulceration, bleeding, fistula formation, and vocal cord paralysis might occur in patients with ZD [4]. 
The mainstay of treatment for symptomatic ZD is surgical management [2]. Conservative management may be applicable to patients with small ZD $(<1 \mathrm{~cm})$ [4]. An open approach is suitable for the treatment of large $\mathrm{ZD}$ and includes cricopharyngeal myotomy alone, or myotomy with diverticulectomy, diverticulopexy or diverticular inversion $[2,4]$. Open surgery for ZD can resolve symptoms in $90 \%$ to $95 \%$ of patients $[2,4]$. An endoscopic approach using a rigid or flexible endoscope is an adequate option to treat $\mathrm{ZD}<5 \mathrm{~cm}$ in diameter; the endoscopic surgery procedure includes myotomy, esophagodiverticulostomy, and diverticulectomy and has a clinical resolution rate of $>90 \%[2,4]$. Considering the underlying mechanisms of ZD, it is believed that myotomy should be part of surgical procedure [4]. Patients with ZD larger than $1 \mathrm{~cm}$ diameter with PSHR on endoscopic examination may be candidates for surgical treatment [8], but treatment guidelines for ZD based on randomized comparative trials have not been established because of the low prevalence of ZD. Accordingly, physicians should consider whether surgical treatment is valid in each case of ZD by considering the severity of dysphagia and risk of aspiration as well as the size of ZD.

In conclusion, we treated a patient with ZD who developed chest discomfort similar to ACS as the primary symptom of ZD. CT findings of air particles adjacent to the hypopharynx suggested the possibility of $\mathrm{ZD}$, and esophagography led us to definite diagnosis. Physicians should know that esophageal diseases, including ZD, may show symptoms mimicking ACS, and not obtaining a detailed clinical history can lead to misdiagnosis.

\section{Acknowledgement}

The authors would like to thank Miss Hisae Kuribara for her secretarial assistance under the financial support funded by Gunma Chuo Hospital.

\section{Grant Support}

None.

\section{Conflicts of Interest}

All authors report no conflicts of interest.

\section{References}

1. AchkarE.Zenker's diverticulum. Dig Dis. 1998;16(3):144151.

2. Law R, Katzka DA, Baron TH. Zenker's Diverticulum. Clin Gastroenterol Hepatol. 2014;12(11):1773-1782; quiz e1111-1772.

3. Stack LB, Munter DW. Foreign bodies in the gastrointestinal tract. Emerg Med Clin North Am. 1996;14(3):493521.

4. Bizzotto A, Iacopini F, Landi R, Costamagna G. Zenker's diverticulum: exploring treatment options. Acta Otorhinolaryngol Ital. 2013;33(4):219-229.

5. Richardson BE, Bastian RW. Videoendoscopic swallowing study for diagnosis of Zenker's diverticuli. Laryngoscope. 1998;108(5):721-724.

6. Gorkem SB, Yikilmaz A, Coskun A, Kucukaydin M. A pediatric case of Zenker diverticulum: imaging findings. Diagn Interv Radiol. 2009;15(3):207-209.

7. Boutou AK, Trigonis I, Pigadas A, Argyropoulou P, Stanopoulos I. Exogenous lipoid pneumonia complicated with mycobacterium infection in a subject with Zenker diverticulum. Ann Acad Med Singapore. 2009;38(2):177-178.

8. Bergeron JL, Long JL, Chhetri DK. Dysphagia characteristics in Zenker's diverticulum. Otolaryngol Head Neck Surg. 2013;148(2):223-228.

9. Spieker MR. Evaluating dysphagia. Am Fam Physician. 2000;61(12):3639-3648.

10. Min YW, Rhee PL. Noncardiac chest pain: update on the diagnosis and management. Korean J Gastroenterol. 2015;65(2):76-84.

11. Erdal U, Mehmet D, Turkay K, Mehmet IA, Ibrahim N, Hasan B. Esophagus perforation and myocardial penetration caused by swallowing of a foreign body leading to a misdiagnosis of acute coronary syndrome: a case report. J Med Case Rep. 2015;9:57. 